\title{
Nutrition as Medicine to Improve Outcomes in Adolescents Sustaining a Sports-related Concussion
}

\author{
Krista Casazza $^{1 *}$ and Erin Swanson ${ }^{2}$ \\ ${ }^{1}$ University of Alabama at Birmingham, Department of Pediatrics, Division of Adolescent Medicine, Birmingham, AL 35233, USA; \\ ${ }^{2}$ University of Alabama at Birmingham, Department of Pediatrics, Division of Rehabilitative Medicine, Birmingham, AL 35233, USA
}

\begin{abstract}
Recognition and diagnosis of sports-related concussion (SRC) among adolescents has significantly increased. In, fact, among high school adolescents, SRC incidence has more than doubled from 2007 to 2014, with recent estimates at approximately 2 per 100 athletes. SRC-related research has also increased; recognition of symptoms that may prolong recovery have been examined, potential biomarkers have been scrutinized, return-to-learn and return-to-play protocols have been developed and honed. However, to date, clinicians and researchers have struggled to find effective interventions to mitigate the significant symptoms after SRC and shorten recovery times. Despite the understood role of the brain as the primary regulator of metabolism, and the well-established metabolic impairments evoked after a concussion, nutrition is often ignored as a core complement to the recovery and rehabilitation process. In this article, we will identify deficiencies and/or inadequacies in nutrients post-concussion and provide support for potential exacerbation of injury and delayed recovery due to inadequate intake of nutrients prior to sustaining an SRC. Additionally, we will discuss the effect of derangement of the metabolic cascade post-concussion, and identify key nutrients, that if supplemented immediately post-injury, could increase neuroprotection, and improve recovery outcomes. Animal and cell culture studies have provided substantial evidence for not only the interrelationship of nutrient adequacy and the adaptation in the metabolic cascade post-concussion on neuroprotection, but also key nutrients that if supplemented immediately post-injury could enhance standard of care with minimal risk.
\end{abstract}

Introduction

Sports-related concussion (SRC), like other acute traumatic brain injuries (TBIs), involves mechanical forces that shear neuronal, glial and endothelial membranes, inducing a complex pathophysiologic process that adversely affects the brain.,2 Collectively, these forces induce a primary response involving neuronal and tissue damage, which is subsequently amplified by a 'secondary

Keywords: Sports-related concussion; Adolescent; Athlete; Nutrition.

Abbreviations: SRC, sports-related concussion; TBI, traumatic brain injury; PUFA, polyunsaturated fatty acid; DHA, decosahexanoic acid; ATP, adenosine triphosphate; BCAAs, branched chain amino acids; CNS, central nervous system; ADP, adenosine diphosphate; CRP, C-reactive protein; ALA, alpha-linoleic acid; VDR, vitamin D receptor; BBB, blood brain barrier; NADPH, nicotinamide adenine dinucleotide phosphate; TCA, tricarboxylic acid; PARP-1, poly-adenosine diphosphate-ribose polymerase-1; GH, growth hormone.

Received: August 14, 2017; Revised: September 25, 2017; Accepted: October 07, 2017

${ }^{*}$ Correspondence to: Krista Casazza, University of Alabama at Birmingham, Department of Pediatrics, Division of Rehabilitative Medicine, $16014^{\text {th }}$ Ave S, CPPI 310 Birmingham, Al 35233, USA. E-mail: kcasazza@peds.uab.edu

How to cite this article: Casazza K, Swanson E. Nutrition as Medicine to Improve Outcomes in Adolescents Sustaining a Sports-related Concussion. Exploratory Research and Hypothesis in Medicine 2017;2(4):122-130. doi: 10.14218/ERHM.2017. 00029 . injury' phase in response to membrane and cellular damage. ${ }^{3}$ This encompasses a cascade of biochemical changes that impairs function of the blood-brain barrier, compromises oxygenation and potentiates further tissue damage. ${ }^{4}$

Targeted patient education and improved protective equipment has led to some decrease in primary injury. ${ }^{5}$ However, to date, the adverse health effects of secondary injury (e.g., sleep disruption, depression, impulsivity, decline in cognitive function, migraine, impaired vision) have not been met with effective treatment. It is generally considered that decline in neuropsychological functioning is transient, resolving to pre-injury benchmarks within 7-10 days, ${ }^{6}$ with the timeline paralleling the acute neurometabolic events triggered by the concussive impact. ${ }^{7}$ However, reports linking the injury to long-term declines in cognitive functioning, ${ }^{8,9} \mathrm{de}-$ pression and mild cognitive impairment has propagated substantial scrutiny of the "transient nature" of SRC, ${ }^{10-12}$ particularly during adolescence.

The injured adolescent brain poses a unique complexity to understanding the pathophysiology of concussion from the incident to the recovery process which extend investigation beyond adult trauma, ${ }^{13}$ rodent models and cell culture towards longitudinal studies assessing the short- and long-term intricate cognitive, emotional, behavioral, neurobiological and neuropathological consequences of concussions that can also identify predictors and modifiers 
of outcomes. ${ }^{14}$ Clinical studies have focused predominantly on descriptive or observational investigations into qualitative symptoms and/or semi-quantitative analysis of cognitive impairments. ${ }^{15}$ As such, important elements of the underlying pathophysiology of concussion have been delineated through experimental models and reports of the long-term effects of concussion in adult athletes (e.g., the National Football League).

Data collected in adults and animals, however, are unable to account for developmental differences and changes in mental status, respectively. For example, Kerr et al. ${ }^{16}$ highlighted a difference in return-to-play times in adolescents demonstrating the unique adaptions and recovery during this critical developmental period and highlighting the need for enhanced management in this population. Given the unique cascade of series of adaptive neurometabolic responses that take place in the brain following a head injury, optimizing treatment strategies-particularly during the critical adolescent brain developmental period-are needed.

We review research evidence for potential benefits of nutrition as medicine in SRC and explore the contention of how nutrient inadequacies affect such. According to this concept, neurometabolic consequences contribute to the development and persistence of post-concussive symptoms after SRC in some youth. We hypothesize that a) nutrition supplementation initiated immediately postSRC represents a key component of rehabilitation with particular relevance in adolescents; and b) the poorer outcomes in females can be explained at least in part by greater inadequacies of nutrients in females relative to their male counterparts prior to sustaining a concussion. Addition of nutrition as medicine to guidelines that emphasizes prompt supplementation is encouraged.

A key pathophysiological phenomenon after a mechanical trauma to the brain is the complex cascade of neurochemical and neurometabolic events and consequent alterations in cerebral metabolism and blood flow, which may result in an energy crisis. ${ }^{7,17,18}$ Two major alterations of glucose metabolism have been described: hyperglycolysis (and thus hyperglycemia) and oxidative dysfunction (rampant activation of the inflammatory cascade). Particularly, glutamate release from damaged cells leads to excitotoxicity, mounting an adverse response including the influx of $\mathrm{Ca}^{2+}$ through glutamate receptors and voltage-gated $\mathrm{Ca}^{2+}$ channels. ${ }^{19,20}$ This in turn activates calcium-dependent proteases, such as calpains and lipases, which degrade membrane phospholipids, leading to the release of fatty acids. ${ }^{7,17,18}$ Fatty acids (primarily arachidonic acid, the precursor of eicosanoids) are released from membranes and transformed into pro-inflammatory eicosanoids which exacerbate tissue injury by inducing vasoconstriction and platelet aggregation, increased inflammation, and production of free radicals. ${ }^{21}$ Within hours, there is also activation of complex dynamics of local cytokine and chemokine production. Concomitantly, synthesis and release of anti-inflammatory factors such as the $\omega-3$ polyunsaturated fatty acid (PUFA) docosahexanoic acid (DHA) are oxidized as fuel; thus, their role as structural components of membranes needed for cellular repairs is attenuated. ${ }^{22}$

The stress response is also known to induce gluconeogenesis and glycogenolysis, as well as the release of cortisol, further exacerbating hyperglycemia and as a consequence augmenting cellular injury and secondary response., ${ }^{7,17,18}$ Increased glutamate release and subsequent increased lactate production (and acidosis) are also associated with hyperglycemia. Although a general characterization of the post-concussion adaptive responses exists, each concussion is unique, with developmental stage, sex and race differences observed in addition to brain region that was affected.

We contend supplementation-specific dietary components alone and/or in combination have the potential to positively influence and to effectively treat the neuroinflammation associated with
SRC. Despite the understood role of the brain as the primary regulator of metabolism and the well-established metabolic impairments evoked after a concussion, nutrition is often ignored as a core complement to the recovery and rehabilitation process. Herein, we will identify deficiencies and/or inadequacies in nutrients post-concussion and provide support for potential exacerbation of injury and delayed recovery due to inadequate intake of nutrients prior to sustaining an SRC.

Specifically, among high school adolescents, SRC incidence has more than doubled from 2007 to $2014,,^{23}$ with recent estimates at approximately 2 per 100 athletes. ${ }^{24}$ Rapid changes in brain organization and development occur in adolescence, with profound reshaping of the prefrontal, parietal temporal-associated, and sensorimotor cortex prior to achieving adult-like connections by the mid-20s. These areas, which do not fully mature until approximately 18 years of age, play a substantial role in executive functions such as problem solving and decision making. ${ }^{14}$ Paralleling the structural changes are changes in cerebral glucose metabolism and cerebral blood flow. ${ }^{25}$

Although post-concussed adolescent athletes often display no gross neural pathologies and have no immediate threat to their life, profound biochemical changes induced by injury in immature, highly-sensitive structures (e.g., the prefrontal cortex, hippocampus, hypothalamus) during this critical developmental stage can increase risk for several neuropsychiatric conditions, with farreaching acute and chronic systemic effects. Brain injury, of any severity, in the developing brain is complicated by ongoing cerebral maturation. ${ }^{26}$ Of crucial importance in prognosis is the rapid increase in reactive stressors that heighten the metabolic demand on the brain. ${ }^{27}$

Concussion induces inflammatory sensory stimulus that is preferentially transmitted to brainstem and specific hypothalamic nuclei which have direct connection to the hypothalamic paraventricular nucleus. Specifically, activation of the inflammatory cascade, increased protein catabolism and altered energy metabolism have been well-established as inducing long-term adverse effects on the developing brain via altered neuroendocrine and physiologic processes. ${ }^{28}$ Increased inflammation markedly affects neurotransmission within emotional regulatory brain circuits and can dysregulate the hypothalamic-pituitary-adrenal axis. Consequently, a strategy that enhances the brain's response against harmful effects of inflammation and protein catabolism may potentiate damaging post-concussive effects in the stress-sensitive brain structures of the adolescent athlete.

Energy (macronutrient) and micronutrient (vitamin and mineral) needs during adolescence are relatively high, as compared to in adulthood. While few studies have evaluated the dietary patterns of young athletes, particularly those participating in sport at the community/high school level and especially based on sex, race/ethnicity and socioeconomic status, it has been reported that dietary intakes in young athletes are reportedly superior to their non-athletic counterparts. ${ }^{29-32}$ However, the increased demands of competition in the context of growth and development imply that the potential consequences of a deficiency may be more detrimental in athletes.

To get a better understanding of how certain nutrients may aid in the treatment of SRC, it is important to understand the neuromolecular cascade that occurs in the brain after a concussion, which has been best studied in animal TBI models. ${ }^{33}$ Observational data consistently show that during the initial post-concussed state, energy and protein deficits are apparent and are associated with worse outcomes. Insufficient micronutrient intakes in the general population adolescent population can profoundly impact energy and macronutrient metabolism, particularly for the B vitamins, vitamin D 
and iron. In this review, we will demonstrate specific nutrients can be utilized for post-SRC supplementation based on their putative roles in the mechanisms of brain injury. ${ }^{33}$

\section{Energy crisis}

In an effort to restore cellular homeostasis and membrane potential, hyperactive adaptive responses of adenosine triphosphate (ATP)requiring ion pumps occur, causing hyperglycolysis and concomitant low intracellular energy reserves. ${ }^{3}$ In the context of reduced cerebral blood flow (due to edema), a mismatch between energy supply and demand ensues. Disturbance in intracellular calcium flux, manifests into sequestration of calcium into mitochondria, which can then result in mitochondrial dysfunction, exacerbate oxidative metabolism and worsen the cellular energy crisis. After an initial period of hyperglycolysis and metabolic uncoupling, glucose metabolic rates go into a state of hypoglycolysis. Energy crisis is associated with behavioral impairments in spatial learning as well as altered gene expression and enzyme/transporter regulation, which may underlie long-term sequelae.

\section{Glucose}

The post-injury energy crisis and glucose metabolic disturbance is exacerbated by increased flux of glucose through the pentose phosphate pathway and inhibition of key glycolytic enzymes. Emerging data suggest that under these post-brain injury conditions of impaired glycolytic metabolism, glucose may not be the best fuel for the injured brain. ${ }^{34-36}$ While the neuroprotective benefit of a ketogenic diet after SRC has yet to be investigated systematically, some studies in animals have demonstrated improvement in contusion volume and behavioral outcomes with a ketogenic diet. ${ }^{37}$

\section{Protein}

Protein and its amino acid building blocks play critical roles in various metabolic and energetic pathways. As a substantial amino acid pool does not exist, protein homeostasis depends upon a functioning system of protein degradation and recycling. ${ }^{38}$ The effects of energy crisis trigger protease activation and apoptotic cell death, limiting protein synthesis. Further, the profound increases in resting energy expenditure in the injured brain and associated negative nitrogen balance induce transient protein inadequacy. ${ }^{39}$

The lack of availability of essential amino acids due to secondary inflammatory cellular damage not only adversely affects cell survival and function in the injured brain but can also induce system-wide detrimental effects. Increased protein catabolism is observed within 24 hours of injury, and low levels of branchedchain amino acids (BCAAs), histidine and methionine have been shown to persist 2 months after injury. ${ }^{40-43}$ Protein catabolism and subsequent hypoalbuminemia can also disrupt mineral availability (e.g., zinc, iron) ${ }^{44}$ further exacerbating oxidative stress ${ }^{45}$ Restoration of protein and nitrogen balance may reduce oxidative stress secondary to concussive injury and facilitate recovery. ${ }^{46}$

Beyond protein synthesis, essential amino acids, provided only through the diet, serve as a substrate for key molecules. For example, BCAAs are integral in overall energy metabolism, regulation of gluconeogenesis and protein synthesis, as well as functioning as a major source of nitrogen for glutamine and nitric oxide production. As such, inadequate protein and BCAA availability in the post-concussed state impacts a number of biological processes, including cytoprotection and gene expression. ${ }^{47}$ While it has been suggested that protein inadequacy may be related to a decreased overall intake following a brain injury, a study of healthy fasted volunteers versus individuals with a traumatic brain injury, in which both groups had not eaten for 7 hours demonstrated significantly greater protein catabolism in TBI. Given that the adolescent athlete requires approximately $1.2-1.5 \mathrm{~g} /$ protein/day to maintain lean body mass and normal protein synthesis, protein supplementation post-concussion is a reasonable strategy to improve outcomes following a SRC.

\section{BCAAs}

In the brain, BCAAs serve as important metabolic precursors for synthesis of proteins and neurotransmitters, including dopamine, serotonin and norepinephrine. ${ }^{48}$ Following brain injury, BCAAs become readily oxidized, potentially contributing to the metabolic crisis post-SRC. Lower BCAAs in TBI patients relative to controls has been observed in various studies. ${ }^{40-43,49}$ BCAA supplementation in rodents has been shown to restore net synaptic efficiency and hippocampal function and consequent restoration of cognitive function. ${ }^{50-52}$

\section{Creatine}

Creatine is well-known for its use in athletes for purported benefits regarding muscle hypertrophy. However, as a biochemical effect in the central nervous system (CNS) via phosphorylation of adenosine diphosphate (ADP) to make ATP, and theoretically decreasing hyperglycolysis and oxidative damage, creatine has emerged as a potential treatment of concussions. ${ }^{53}$ The primary role of creatine in the body involves energy homeostasis via maintenance of constant cellular ATP levels in tissues with highly fluctuating energy demands (i.e. muscle and brain). ${ }^{54,55}$ The creatine-ATP energy system enables rapid, efficient ATP regeneration in the absence of oxygen. The system decreases glycolysis and the consequential synthesis and accumulation of lactic acid.

Assessment of creatine pre- and post-concussion by magnetic resonance spectroscopy has shown decreased levels in the brain after sustaining a concussion. ${ }^{56}$ A potential antioxidant effect has also been hypothesized. In animal models, creatine supplementation pre-TBI has demonstrated a neuroprotective effect on mitochondrial function and secondary injury. ${ }^{57}$ Human studies, though limited to severe TBI, have shown increased cognitive function and decreased headache and dizziness. ${ }^{58}$

\section{$D H A$}

PUFAs, specifically $\omega-3$, are essential for the rapid neurogenesis during brain development as well as neuroprotection across the lifespan. ${ }^{59}$ Dietary depletion has been shown across species to impair structural and neuronal development and function in early life. ${ }^{60}$ In addition to the structural role, $\omega-3$ PUFAs, particularly DHA, are involved in multiple brain functions, including cell membrane fluidity, receptor affinity and modulation of signal transduction molecules. ${ }^{61-63}$ The mechanisms involved in $\omega-3$ PUFAs' neuroprotection over the life course (including injury) include decreased neuroinflammation and oxidative stress, neurotrophic support and activation of cell survival pathways. The level of total $\omega-3$ PUFAs in plasma is inversely correlated with the level of pro-inflammatory markers (IL-6, TNF $\alpha$, IL-1 and C-reactive 
protein (CRP)) and the $\omega-6 / \omega-3$ PUFA ratio is negatively correlated with IL-10 anti-inflammatory marker.

$\omega-3$ PUFAs are synthesized through desaturation and elongation reactions from their precursor, an 18-carbon-atom fatty acid, alphalinolenic acid (ALA), which is available in the diet. ${ }^{64}$ However, the $\omega-3$ PUFA required by the CNS is essential, and not produced de novo in mammals. Under pathological conditions, including SRC, phospholipases rapidly release PUFAs from membranes. ${ }^{59}$ The accumulation of free fatty acids activates inflammatory pathways through cell-specific receptors and activation of protein kinases. In turn, a large number of lipid mediators augment oxidative stress and induce mitochondrial dysfunction. ${ }^{65} \mathrm{DHA}$, the primary structural $\omega-3$ PUFA present in the brain, preferentially accumulates in the frontal cortex and hippocampus, constituting up to $97 \%$ of the $\omega-3$ PUFAs in the brain. It is converted to oxylipin intermediates, thus rendering high CNS demands for DHA unattainable. ${ }^{65}$ The unavailability of DHA in such instances as TBI, affects physical properties of membranes via alteration of transmembrane enzymes and binder receptor proteins as well as neurotransmitter synthesis and release. ${ }^{66}$ Substantial evidence for animal studies investigating $\omega$-3 PUFA supplementation before or after TBI supports a protective effect of $\omega-3$ PUFA on the brain by limiting structural damage to the axon and attenuating events inducing neuronal apoptosis.

While an adequate supply of DHA is recommended, worldwide consumption is low, with most adolescents having an inadequate intake of $\omega-3$ PUFA ${ }^{67}$ Dietary sources of DHA are limited, with cold-water algae being the primary producers of DHA and EPA. Fish are also rich sources of DHA due to a diet consisting of algae. Dietary intake of the essential FA and precursor to DHA $\alpha$-linolenic acid (ALA; 18:3n-3) is generally much higher. ${ }^{68,69}$ Although ALA can be metabolically converted to DHA, the conversion rate is low. Further, diets higher in ALA seem to limit the conversion rate by increasing the rate of ALA oxidation, ${ }^{70}$ and contemporary food manufacturing has dramatically altered the $\omega-3 / \omega-6$ PUFA ratio in the Western diet. There has been an increase in $\omega-6$ PUFA intake and concomitant decrease in $\omega-3$ PUFA intake.

In humans, dietary supplementation with DHA against acute and chronic inflammation within the CNS was first reported as case studies (i.e. lone survivor of the Sago Mine disaster, TBI after a motor vehicle accident in an adolescent), in which recovery appeared to be associated with supplementation of PUFAs. Supplementation of DHA has been shown to reduce glutamate-induced excitotoxicity and both axonal and neuronal injury through modulation of ion channels. In embryonic hippocampal and cortical neuron cultures, supplementation with DHA has been shown to increase neurite number and length. ${ }^{71}$ In rodent models, DHA supplementation post-moderate TBI was shown to decrease number of axon apoptosis and to increase hippocampal neurons as well as decrease pro-inflammatory cytokines and markers of oxidative stress. ${ }^{71-76}$

Rodent models have also been fairly consistent in demonstrating a beneficial effect of DHA supplementation through multiple signaling pathways in brain injury, spinal cord injury and cardiac ischemia-reperfusion. ${ }^{65}$ Specifically in models of head injury, prophylactic supplementation with DHA attenuates white matter damage, as evidenced by fewer beta amyloid precursor-positive axons, enhanced preservation of myelin, and protection of neurofilament morphology. ${ }^{73,77-80}$ Further, DHA supplementation has been shown to allay glutamate cytotoxicity, suppress mitochondrial dysfunction and the eventual development oxidative stress, decrease calcium influx, and down-regulate $\alpha$-amino-3-hydroxy5-methyl-4-isoxazolepropionic acid receptor subunits. ${ }^{81}$ While the preservation of white matter undoubtedly aids in maintaining neurocognitive function following injury, the blunting of injuryinduced reductions in molecular elements important for learning may also play a role..$^{82}$

While the precise mechanism by which DHA confers a neuroprotective effect is multifaceted and not completely understood, it likely includes a series of mutual mechanisms that enhance the structural integrity of neurons, thereby diminishing the secondary pathological sequelae (i.e. neuroinflammation, impaired energy metabolism, glutamate release) that occurs post-injury. In summary, $\omega-3$ PUFAs have been shown to address several of the hallmark pathologic features of brain injury, such as excitotoxicity, oxidative stress, and inflammation. A dose-dependent relationship exists, whereby plasma phospholipid DHA concentrations increase up to a dosage of approximately $2 \mathrm{~g} /$ day, after which any further increase in dose negligibly increases plasma phospholipid concentration. ${ }^{67}$ However, despite substantial evidence from rodent models of TBI, human-based studies examining the neuroprotective effects of DHA have been limited to clinical case studies.

\section{Vitamin D}

Vitamin D, most notably studied for its role in calcium homeostasis, has emerged as a significant contributor to a broad range of physiologic activities, including muscle function, metabolic control and immune modulation. More recently, it has been identified as a substance with substantial involvement in brain development, health and function. ${ }^{83}$ The vitamin D receptor (VDR) protein is reportedly expressed broadly in the brain, including the neurons and glial cells. ${ }^{84}$ Further, $1,25(\mathrm{OH}) \mathrm{D}$ appears to influence neuronal cell differentiation and exert neuroprotective actions against cytotoxicity. Vitamin D deficiency exacerbates inflammatory response and cell death. ${ }^{83}$

The role of vitamin D supplementation post-TBI and/or investigation of the role of vitamin D deficiency on outcomes following TBI was recently reviewed. ${ }^{83}$ A significant reduction in phosphorylation of nuclear factors, which in turn stimulate downstream genes involved in the inflammatory cascade, were shown to be reduced by vitamin D treatment post-TBI ${ }^{85}$ In separate studies, Tang et al. ${ }^{86}$ and Cekic et al. ${ }^{87}$ demonstrated that vitamin D deficiency was associated with more adverse pathophysiological outcomes following TBI. Of note, an estimated $40 \%$ of adolescents have insufficient vitamin D. ${ }^{88}$ Further, in a clinical sample, Hua et $a l .{ }^{89}$ observed improved functional outcomes with vitamin D supplementation post-TBI.

Despite its emergence as an integral cofactor in brain health, there have been no clinical trials to assess the efficacy of vitamin $\mathrm{D}$ as a treatment for mild TBI. Studies conducted in other aspects of neurocognition (i.e. stroke, Alzheimer's, dementia) have established an association with vitamin D insufficiency and suggest prevention of vitamin D deficiency may serve a valuable role as a neuroprotective therapy. ${ }^{45,90,91}$

\section{Thiamin}

Restoration of energy metabolism and membrane homeostasis may reduce oxidative stress secondary to concussive injury and facilitate recovery. "Much of the role in neuronal excitability has been derived from the evaluation of effects of alcohol on thiamine diphosphate in the context of the brain and Wernike-Korsakoff syndrome. The synergistic effects of thiamin deficiency and alcohol-induced neurotoxicity from excessive glutamate release elicit acute cerebral damage and subsequent irreversible neuronal dam- 
age." ${ }^{92}$ It is biochemically analogous that, in TBI, changes due to inadequate thiamin primarily involve decreased activity of the enzymes, a process that leads to energy deficits as a consequence of impairment of the tricarboxylic acid (TCA) cycle which can ultimately lead to neuronal apoptosis.

Altered energy metabolism, secondary to unavailability of thiamin co-factors, also results in the development of lactic acidosis in response to impaired TCA cycle activity. This acidosis can contribute to cytotoxic brain edema, a contributor to excitotoxicity, as well as a net increase in free radical production and oxidative stress along with development of inflammatory processes. Because thiamin represents an integral cofactor involved in processes associated with the metabolism of lipids, glucose, amino acids and neurotransmitters, inadequate levels can lead to severe complications in the nervous system. ${ }^{93}$ Diminished availability of thiamin in the brain profoundly limits enzyme activity, leading to alterations in mitochondrial activity, impairment of oxidative metabolism, decreased energy status, and exacerbation of secondary injury pathways. $^{93}$

Accumulating evidence indicates that deficiency in thiamin induces mild chronic impairment of oxidative metabolism of microglia, astrocytes and endothelial cells, as well as abnormalities of cerebral glucose metabolism. This in turn, increases neuronal apoptosis. In animal models, thiamin inadequacy is associated with pathology of brain injury. ${ }^{94}$ Compromised mitochondrial function, is also apparent and leads to increased free radical production that contributes to neuronal dysfunction and apoptosis. Further, inadequate thiamin dysregulates glucose metabolism, which induces alterations in cerebral blood flow, along with compensatory stimulation of lactic acidosis, which contributes to impairments in the blood-brain barrier (BBB) breakdown. BBB disruption then leads to decreased fatty acid synthesis, which itself can lead to demyelination and decreased nucleic acid synthesis. ${ }^{93}$

\section{Niacin}

Niacin (nicotinamide; vitamin B3) is regarded as a broad-spectrum neuroprotectant. Its role is as a precursor for $\mathrm{NAD}+$, the coenzyme commonly known for its role in the electron transport chain, allowing for the production of ATP. ${ }^{45}$ Activation of microglia in TBI causes up-regulation of nicotinamide adenine dinucleotide phosphate (NADPH) oxidase and activation of the inflammatory cascade and consequent cytotoxicity. Nicotinamide supplementation has been shown to attenuate secondary damage in TBI. ${ }^{95}$

The mechanistic underpinnings of the purported benefits have been linked to increased bioavailability of ATP to cells following the neural insult, an inhibition of injury-induced poly-adenosine diphosphate-ribose polymerase-1 (PARP-1) and sirtuin-1 activation, both of which act to deplete NAD+ and reductions in apoptotic and necrotic death. The decrease in NAD+ is that which is often linked to apoptosis. NAD + theoretically provides cytoprotection through pathways that involve poly(ADP-ribose) polymerase, Akt, mitochondrial membrane potential and cysteine protease activity, and prevents apoptosis by maintaining DNA integrity as well as protects against microglial activation with subsequent phagocytic destruction of cells. ${ }^{96}$ Niacin is also actively involved in redox reactions, modulates the mitochondrial permeability transition pore and replenishes nicotinamide adenine dinucleotide phosphate levels with resultant increases in glutathione reducing free radicals produced following injury.

Direct and indirect inhibition of PARP-1, has also been reported to have beneficial effects on tissue and behavior following injury, while activation of the PARP-1 pathway has been shown to be detrimental. In clinical trials, nicotinamide supplementation has been reported to significantly reduce injury volume, decrease glial fibrillary acidic protein activation, reduce the blood brain barrier breach, reduce acute edema, reduce behavioral impairments, and improve outcomes. ${ }^{97}$ Additionally, niacin treatment has been shown to counteract gene expression changes due to TBI via down-regulation of genes activated by injury.

\section{Iron}

The increased need for iron in adolescents to support growth, expansion of red blood cell volume and addition of lean body mass have been long appreciated. Iron is important to maintain adequate hemoglobin concentrations as well as total iron stores during growth. Furthermore, it is well documented that iron deficiency anemia can lead to fatigue, performance, and learning ability. ${ }^{98,99}$ Brain injury is associated with iron deficiency and has been shown to exacerbate symptoms of fatigue and muscle weakness. ${ }^{100}$ In turn, iron deficiency impairs erythropoiesis and enhances eryptosis, resulting in anemia. Improper distribution of iron within the body has been suggested to contribute to the iron deficit in the erythropoietic system and iron overload in the substantia nigra in neuroinflammatory conditions, such as Parkinson's disease. ${ }^{101}$ Moreover, iron modulates dopamine synthesis and re-uptake (as does vitamin $\mathrm{C}$ at the level of dopamine $\beta$-hydroxylase); tyrosine hydroxylase, an enzyme responsible for dopamine synthesis, is iron-dependent. Iron deficiency has also been shown to impair dopamine re-uptake in rodents. ${ }^{102}$

\section{Sex differences in concussion outcomes}

Although arguably confounded by across-sport participation numbers and inconsistencies in baseline measures, sex differences in both the incidence and outcomes of concussions have been reported. ${ }^{103,104}$ Collectively, in evaluations of sports played by both sexes (e.g., soccer, baseball/softball, basketball), concussions represented a greater proportion of total injuries in females, with a greater number and severity of symptoms, poorer outcomes, and longer duration to recover. ${ }^{103,105-111}$ The mechanisms underlying this sex difference are unclear, and may in part relate to biological differences that are compounded by cultural distinctions in how concussions are reported and managed. Proposed differences in neck strength, movement biomechanics and symptom reporting prevalence in females compared with males have been suggested. However, we contend dietary factors likely play a substantial role.

Nutrition parameters in adolescent athletes are sparse, with the majority focused at the college level. In female collegiate athletes, Sato et al. ${ }^{112}$ reported blood thiamin concentration decreased significantly during training, with a greater magnitude in females. Iron balance of the female adolescent athletes, beyond the traditional explanation of dietary intake/iron loss balance, has emerged as a significant concern. A recent study among collegiate athletes observed $2.2 \%$ iron deficiency anemia and $30.9 \%$ iron deficiency without anemia in females, as compared to $1.2 \%$ and $2.9 \%$, respectively, in males. ${ }^{113}$ Further, Sandstrom and colleagues recently reported lower serum iron and elevated hepcidin in the athlete group relative to that in the non-athlete group. ${ }^{114}$

Impaired iron balance also influences metabolic fuel availability and associated growth hormone (GH) function. ${ }^{114}$ In addition, inadequate iron suppresses ghrelin concentrations. Moreover, iron deficiency coupled with impaired GH function observed in SRC may reduce free fatty acid availability to further lower serum glu- 
cose concentrations and enhance muscle protein degradation. ${ }^{115}$

Key points

- Although impaired glucose metabolism is characteristics of SRC under post-brain injury conditions of impaired glycolytic metabolism, glucose may not be the best fuel for the injured brain.

- Protein is essential for normal brain energy metabolism. Inadequacy leads to a wide-ranging cascade of events, many of which overlap, and may interact, ultimately resulting in neuronal apoptosis.

- Given the consistent reporting of depressed endogenous levels and absence of adverse outcomes from supplementation of BCAAs, supplementing appears feasible.

- A potential synergistic effect of creatine supplementation post-SRC on muscular and cognitive function warrants investigation.

- Animals supplemented with $\omega-3$ PUFAs consistently exhibit enhanced resilience to TBI, with functional outcomes mirroring those biological indicators of injury, even following multiple mild TBIs, similar to that which would be observed in repetitive sports-related concussive injuries over a lifetime of play.

- Thiamin is essential for normal brain energy metabolism. Inadequacy leads to a wide-ranging cascade of events, many of which overlap, and may interact, but ultimately result in neuronal apoptosis.

- Evidence for improved outcomes using vitamin D supplementation, particularly in those who are deficient, lends credence to the potential for use to limit complications.

\section{Future directions/prospective/prediction}

Because of the complex and multifactorial nature of the adverse effects of the TBI process, identifying a single strategy that will be sufficient to address the sequelae of physical, behavioral and cognitive manifestations is unlikely. However, capitalizing on the multitude of beneficial effects of dietary components represents a cost- effective strategy for reducing both the cognitive and behavioral deficits. Further study in humans is needed to demonstrate the utility of post-concussive supplementation for cognitive and behavioral recovery after mild TBI.

While nutritional intervention has been shown to change metabolic function in cell culture, brain function in animal models of mild TBI as well as other neurological populations, some important unanswered questions remain. For example, with similar severity of injury, why are complications more severe among some individuals? Will baseline nutritional status prior to concussion influence response to treatment? We hypothesize that the differences in response to and recovery from concussion include physiologic as well as environmental factors.

Though improving treatment post-concussion has received recent attention, lack of baseline data describing the underlying processes limits identification of a successful approach to improve long-term outcomes and elucidate differences in complications between sexes as well as emerging differences among racial groups. Future studies will require a pre-participation baseline assessment in student athletes. Given the intense consequences of lack of fuel availability for recovery of the injured brain, with little information on the nutritional needs in the still developing adolescent brain, evaluation of baseline nutritional status (i.e. energy metabolism, essential nutrient adequacy) is required.

\section{Conclusions}

Treatment strategies of adolescents sustaining an SRC often include principles, guidelines and recommendations developed for adults. A number of unique concerns remain with respect to the developing brain, including the greater susceptibility to long-term sequelae. The sudden and profound "neurotoxicity" induced by biochemical changes following SRC manifest energy crisis, mitochondrial dysfunction, activation of the inflammatory cascade, and metabolic vulnerability to nutritional inadequacies. Prompt initiation of nutritive therapy represents an integral facets of concussion management. Concussive outcomes may differ in terms of the precise underlying biochemical anomalies and functional impairments, and by sex.

Current best-practice guidelines recommend a period of cognitive and physical rest, followed by a gradual return to school and activity, with little (if any) focus on restoring metabolic sufficiency. The concept of physical and cognitive rest as the cornerstone of concussion management asserts that during the acute (1-7 days, which are arguable longer in youth) post-injury period of increased metabolic demand and limited ATP reserves, non-essential activity draws oxygen and glycogen away from injured neurons. This "shut down" or "dark closet" approach following concussion ignores nutrition as a key component of therapy.

Nutrient adequacy is essential for compensatory metabolic regulation, yet is often overlooked as a critical component to metabolic recovery after concussion. Sufficiency of endogenous substrates is a strong determinant of recovery and rehabilitation; thus, nutrition supplementation represents a viable and valuable component of treatment, particularly in adolescents post-SRC.

\section{Conflict of interest}

The authors have no conflict of interest(s) related to this publication.

\section{Author contributions}

Conception and/or the acquisition of literature reviewed (KC, $\mathrm{ES})$, interpretation of the overall concepts (KC, ES), drafting of the manuscript $(\mathrm{KC}, \mathrm{ES})$, revising or questioning its intellectual content $(\mathrm{KC}, \mathrm{ES})$, and giving approval of the final version of the manuscript for publication (KC, ES).

\section{References}

[1] Harmon KG, Drezner JA, Gammons M, Guskiewicz KM, Halstead M, Herring SA, et al. American Medical Society for Sports Medicine position statement: concussion in sport. Br J Sports Med 2013;47(1):1526. doi:10.1136/bjsports-2012-091941.

[2] Steenerson K, Starling AJ. Pathophysiology of sports-related concussion. Neurol Clin 2017;35(3):403-408. doi:10.1016/j.ncl.2017.03. 011.

[3] Laskowski RA, Creed JA, Raghupathi R. Frontiers in neuroengineering pathophysiology of mild TBI: implications for altered signaling pathways. In: Kobeissy FH, ed. Brain Neurotrauma: Molecular, Neuropsychological, and Rehabilitation Aspects. Boca Raton (FL): CRC Press/ 
Taylor \& Francis (c) 2015 by Taylor \& Francis Group, LLC.; 2015.

[4] Ling $\mathrm{H}, \mathrm{Hardy} J$, Zetterberg $\mathrm{H}$. Neurological consequences of traumatic brain injuries in sports. Mol Cell Neurosci 2015;66(Pt B):114-122. doi:10.1016/j.mcn.2015.03.012.

[5] Rowson S, Bland ML, Campolettano ET, Press JN, Rowson B, Smith JA, et al. Biomechanical perspectives on concussion in sport. Sports Med Arthrosc 2016;24(3):100-107. doi:10.1097/JSA.0000000000000121.

[6] McCrory P, Meeuwisse WH, Aubry M, Cantu RC, Dvořák J, Echemendia RJ, et al. Consensus statement on concussion in sport: the 4th International Conference on Concussion in Sport, Zurich, November 2012. J Athl Train 2013;48(4):554-575. doi:10.4085/1062-605048.4.05.

[7] Giza CC, Hovda DA. The new neurometabolic cascade of concussion. Neurosurgery 2014;75(Suppl 4):S24-S33. doi:10.1227/ NEU.0000000000000505.

[8] Moser RS, Schatz P. A case for mental and physical rest in youth sports concussion: it's never too late. Front Neurol 2012;3:171. doi:10.3389/fneur.2012.00171.

[9] Henry LC, Tremblay S, De Beaumont L. Long-term effects of sports concussions: bridging the neurocognitive repercussions of the injury with the newest neuroimaging data. Neuroscientist 2017;23(5):567578. doi:10.1177/1073858416651034.

[10] Guskiewicz KM, Marshall SW, Bailes J, McCrea M, Harding HP Jr, Matthews A, Mihalik JR, et al. Recurrent concussion and risk of depression in retired professional football players. Med Sci Sports Exerc 2007;39(6):903-909. doi:10.1249/mss.0b013e3180383da5.

[11] Kerr ZY, Marshall SW, Harding HP Jr, Guskiewicz KM. Nine-year risk of depression diagnosis increases with increasing self-reported concussions in retired professional football players. Am J Sports Med 2012;40(10):2206-2212. doi:10.1177/0363546512456193.

[12] Guskiewicz KM, Marshall SW, Bailes J, McCrea M, Cantu RC, Randolph $\mathrm{C}$, et al. Association between recurrent concussion and late-life cognitive impairment in retired professional football players. Neurosurgery 2005;57(4):719-726; discussion 719-726. doi:10.1093/neurosurgery/57.4.719.

[13] Haider MN, Leddy JJ, Pavlesen S, Kluczynski M, Baker JG, Miecznikowski JC, et al. A systematic review of criteria used to define recovery from sport-related concussion in youth athletes. $\mathrm{Br} J$ Sports Med 2017; pii: bjsports-2016-096551. doi:10.1136/bjsports2016-096551.

[14] Sports-related concussions in youth: improving the science, changing the culture. Mil Med 2015;180(2):123-125. doi:10.7205/MILMEDD-14-00516.

[15] Clark MD, Asken BM, Marshall SW, Guskiewicz KM. Descriptive characteristics of concussions in national football league games, 2010-2011 to 2013-2014. Am J Sports Med 2017;45(4):929-936. doi:10.1177/0363546516677793.

[16] Kerr ZY, Zuckerman SL, Wasserman EB, Covassin T, Djoko A, Dompier TP. Concussion symptoms and return to play time in youth, high school, and college american football athletes. JAMA Pediatr 2016;170(7):647-653. doi:10.1001/jamapediatrics.2016.0073.

[17] Giza CC, Kutcher JS. An introduction to sports concussions. Continuum (Minneap Minn) 2014;20(6 Sports Neurology):1545-1551. doi:10.1212/01.CON.0000458975.78766.11.

[18] Hovda DA. The neurophysiology of concussion. Prog Neurol Surg 2014;28:28-37. doi:10.1159/000358749.

[19] Henry LC, Tremblay S, Boulanger Y, Ellemberg D, Lassonde M. Neurometabolic changes in the acute phase after sports concussions correlate with symptom severity. J Neurotrauma 2010;27(1):65-76. doi:10.1089/neu.2009.0962.

[20] Chamard E, Henry L, Boulanger Y, Lassonde M, Theoret H. A followup study of neurometabolic alterations in female concussed athletes. J Neurotrauma 2014;31(4):339-345. doi:10.1089/neu.2013.3083.

[21] Trojian TH, Wang DH, Leddy JJ. Nutritional supplements for the treatment and prevention of sports-related concussion-evidence still lacking. Curr Sports Med Rep 2017;16(4):247-255. doi:10.1249/ JSR.0000000000000387.

[22] Barrett EC, McBurney MI, Ciappio ED. $\omega$-3 fatty acid supplementation as a potential therapeutic aid for the recovery from mild traumatic brain injury/concussion. Adv Nutr 2014;5(3):268-277. doi:10.3945/ an.113.005280.
[23] Zhang AL, Sing DC, Rugg CM, Feeley BT, Senter C. The rise of concussions in the adolescent population. Orthop J Sports Med 2016;4(8):2325967116662458. doi:10.1177/2325967116662458.

[24] Marshall SW, Guskiewicz KM, Shankar V, McCrea M, Cantu RC. Epidemiology of sports-related concussion in seven US high school and collegiate sports. Inj Epidemiol 2015;2(1):13. doi:10.1186/s40621015-0045-4.

[25] Udomphorn Y, Armstead WM, Vavilala MS. Cerebral blood flow and autoregulation after pediatric traumatic brain injury. Pediatr Neurol 2008;38(4):225-234. doi:10.1016/j.pediatrneurol.2007.09.012.

[26] Deshpande SK, Hasegawa RB, Rabinowitz AR, Whyte J, Roan CL, Tabatabaei $A$, et al. Association of playing high school football with cognition and mental health later in life. JAMA Neurol 2017;74(8):909918. doi:10.1001/jamaneurol.2017.1317.

[27] Davis GA, Anderson V, Babl FE, Gioia GA, Giza CC, Meehan W, et al. What is the difference in concussion management in children as compared with adults? A systematic review. Br J Sports Med 2017;51(12):949-957. doi:10.1136/bjsports-2016-097415.

[28] Bigler ED, Abildskov TJ, Goodrich-Hunsaker NJ, Black G, Christensen $\mathrm{ZP}$, Huff $\mathrm{T}$, et al. Structural neuroimaging findings in mild traumatic brain injury. Sports Med Arthrosc 2016;24(3):e42-e52. doi:10.1097/ JSA.0000000000000119.

[29] Manore MM, Brown K, Houtkooper L, Jakicic J, Peters JC, Smith Edge $\mathrm{M}$, et al. Energy balance at a crossroads: translating the science into action. Med Sci Sports Exerc 2014;46(7):1466-1473. doi:10.1249/ MSS.0000000000000318.

[30] Meyer NL, Manore MM, Helle C. Nutrition for winter sports. J Sports Sci 2011;29(Suppl 1):S127-S136. doi:10.1080/02640414.2011.5747 21.

[31] Manore MM. Exercise and the Institute of Medicine recommendations for nutrition. Curr Sports Med Rep 2005;4(4):193-198. doi:10.1007/s11932-005-0034-4.

[32] Manore MM. Nutritional needs of the female athlete. Clin Sports Med 1999;18(3):549-563.

[33] Bolouri H, Zetterberg H. Kobeissy FH. Frontiers in neuroengineering animal models for concussion: molecular and cognitive assessmentsrelevance to sport and military concussions. Brain Neurotrauma: Molecular, Neuropsychological, and Rehabilitation Aspects 2015;Boca Raton (FL)CRC Press/Taylor \& Francis (c) 2015 by Taylor \& Francis Group, LLC.

[34] Wolahan SM, Prins ML, McArthur DL, Real CR, Hovda DA, Martin NA, et al. Influence of glycemic control on endogenous circulating ketone concentrations in adults following traumatic brain injury. Neurocrit Care 2017;26(2):239-246. doi:10.1007/s12028-016-0313-3.

[35] Prins ML, Matsumoto J. Metabolic Response of Pediatric Traumatic Brain Injury. J Child Neurol 2016;31(1):28-34. doi:10.1177/088307 3814549244.

[36] Prins M. Diet, ketones, and neurotrauma. Epilepsia 2008;49(Suppl 8):111-113. doi:10.1111/j.1528-1167.2008.01852.x.

[37] Prins ML, Matsumoto JH. The collective therapeutic potential of cerebral ketone metabolism in traumatic brain injury. J Lipid Res 2014;55(12):2450-2457. doi:10.1194/jlr.R046706.

[38] Thomas DT, Erdman KA, Burke LM. Position of the Academy of Nutrition and Dietetics, Dietitians of Canada, and the American College of Sports Medicine: nutrition and athletic performance. J Acad Nutr Diet 2016;116(3):501-528. doi:10.1016/j.jand.2015.12.006.

[39] Aquilani R, Viglio S, ladarola P, Guarnaschelli C, Arrigoni N, Fugazza $\mathrm{G}$, et al. Peripheral plasma amino acid abnormalities in rehabilitation patients with severe brain injury. Arch Phys Med Rehabil 2000;81(2):176-181. doi:10.1016/S0003-9993(00)90137-0.

[40] Boselli M, Aquilani R, Baiardi P, Dioguardi FS, Guarnaschelli C, Achilli $\mathrm{MP}$, et al. Supplementation of essential amino acids may reduce the occurrence of infections in rehabilitation patients with brain injury. Nutr Clin Pract 2012;27(1):99-113. doi:10.1177/0884533611431068.

[41] Aquilani R, Sessarego P, ladarola P, Barbieri A, Boschi F. Nutrition for brain recovery after ischemic stroke: an added value to rehabilitation. Nutr Clin Pract 2011;26(3):339-345. doi:10.1177/0884533611405793.

[42] Aquilani R, Boselli M, Boschi F, Viglio S, ladarola P, Dossena M, et al. Branched-chain amino acids may improve recovery from a vegetative or minimally conscious state in patients with traumatic brain 
injury: a pilot study. Arch Phys Med Rehabil 2008;89(9):1642-1647. doi:10.1016/j.apmr.2008.02.023.

[43] Aquilani R, ladarola P, Contardi A, Boselli M, Verri M, Pastoris O, et al. Branched-chain amino acids enhance the cognitive recovery of patients with severe traumatic brain injury. Arch Phys Med Rehabil 2005;86(9):1729-1735. doi:10.1016/j.apmr.2005.03.022.

[44] McClain CJ, Twyman DL, Ott LG, Rapp RP, Tibbs PA, Norton JA, et al. Serum and urine zinc response in head-injured patients. J Neurosurg 1986;64(2):224-230. doi:10.3171/jns.1986.64.2.0224.

[45] Scrimgeour AG, Condlin ML. Nutritional treatment for traumatic brain injury. J Neurotrauma 2014;31(11):989-999. doi:10.1089/neu. 2013.3234.

[46] Jeter CB, Hergenroeder GW, Ward NH 3rd, Moore AN, Dash PK. Human traumatic brain injury alters circulating L-arginine and its metabolite levels: possible link to cerebral blood flow, extracellular matrix remodeling, and energy status. J Neurotrauma 2012;29(1):119-127. doi:10.1089/neu.2011.2029.

[47] Dash PK, Hergenroeder GW, Jeter CB, Choi HA, Kobori N, Moore AN. Traumatic brain injury alters methionine metabolism: implications for pathophysiology. Front Syst Neurosci 2016;10:36. doi:10.3389/ fnsys.2016.00036.

[48] Cole JT, Sweatt AJ, Hutson SM. Expression of mitochondrial branchedchain aminotransferase and alpha-keto-acid dehydrogenase in rat brain: implications for neurotransmitter metabolism. Front Neuroanat 2012;6:18. doi:10.3389/fnana.2012.00018.

[49] Jeter CB, Hergenroeder GW, Ward NH 3rd, Moore AN, Dash PK. Human mild traumatic brain injury decreases circulating branchedchain amino acids and their metabolite levels. J Neurotrauma 2013;30(8):671-679. doi:10.1089/neu.2012.2491.

[50] Cole JT, Mitala CM, Kundu S, Verma A, Elkind JA, Nissim I, et al. Dietary branched chain amino acids ameliorate injury-induced cognitive impairment. Proc Natl Acad Sci U S A 2010;107(1):366-371. doi:10.1073/pnas.0910280107.

[51] Elkind JA, Lim MM, Johnson BN, Palmer CP, Putnam BJ, Kirschen MP, et al. Efficacy, dosage, and duration of action of branched chain amino Acid therapy for traumatic brain injury. Front Neurol 2015;6:73. doi:10.3389/fneur.2015.00073

[52] Sharma B, Lawrence DW, Hutchison MG. Branched chain amino acids (BCAAs) and traumatic brain injury: a systematic review. J Head Trauma Rehabil 2017. doi:10.1097/HTR.0000000000000280.

[53] Beni SM, Kohen R, Reiter RJ, Tan DX, Shohami E. Melatonin-induced neuroprotection after closed head injury is associated with increased brain antioxidants and attenuated late-phase activation of NF-kappaB and AP-1. FASEB J 2004;18(1):149-151. doi:10.1096/fj.03-0323fje.

[54] Dean PJ, Sato JR, Vieira G, McNamara A, Sterr A. Multimodal imaging of mild traumatic brain injury and persistent postconcussion syndrome. Brain Behav 2015;5(1):45-61. doi:10.1002/brb3.292.

[55] Dean PJ, Otaduy MC, Harris LM, McNamara A, Seiss E, Sterr A Monitoring long-term effects of mild traumatic brain injury with magnetic resonance spectroscopy: a pilot study. Neuroreport 2013;24(12):677-681. doi:10.1097/WNR.0b013e3283637aa4.

[56] Vagnozzi R, Signoretti S, Floris R, Marziali S, Manara M, Amorini $\mathrm{AM}$, et al. Decrease in $\mathrm{N}$-acetylaspartate following concussion may be coupled to decrease in creatine. J Head Trauma Rehabi 2013;28(4):284-292. doi:10.1097/HTR.0b013e3182795045.

[57] Miao Q, Wang S, Miao S, Wang J, Xie Y, Yang Q. Cardioprotective effect of polydatin against ischemia/reperfusion injury: roles of protein kinase C and mito K(ATP) activation. Phytomedicine 2011;19(1):812. doi:10.1016/j.phymed.2011.06.023.

[58] Genius J, Geiger J, Bender A, Moller HJ, Klopstock T, Rujescu D. Creatine protects against excitoxicity in an in vitro model of neurodegeneration. PLoS One 2012;7(2):e30554. doi:10.1371/journal. pone.0030554.

[59] Denis I, Potier B, Vancassel S, Heberden C, Lavialle M. Omega-3 fatty acids and brain resistance to ageing and stress: body of evidence and possible mechanisms. Ageing Res Rev 2013;12(2):579-594. doi:10.1016/j.arr.2013.01.007.

[60] Weiser MJ, Butt CM, Mohajeri MH. Docosahexaenoic acid and cognition throughout the lifespan. Nutrients 2016;8(2):99. doi:10.3390/ nu8020099.

[61] Salem N Jr, Litman B, Kim HY, Gawrisch K. Mechanisms of action of docosahexaenoic acid in the nervous system. Lipids 2001;36(9):945959. doi:10.1007/s11745-001-0805-6.

[62] Ryan AS, Astwood JD, Gautier S, Kuratko CN, Nelson EB, Salem N Jr. Effects of long-chain polyunsaturated fatty acid supplementation on neurodevelopment in childhood: a review of human studies. Prostaglandins Leukot Essent Fatty Acids 2010;82(4-6):305-314. doi:10.1016/j.plefa.2010.02.007

[63] Kuratko CN, Barrett EC, Nelson EB, Salem N Jr. The relationship of docosahexaenoic acid (DHA) with learning and behavior in healthy children: a review. Nutrients 2013;5(7):2777-2810. doi:10.3390/ nu5072777.

[64] Zarate R, El Jaber-Vazdekis N, Tejera N, Perez JA, Rodriguez C. Significance of long chain polyunsaturated fatty acids in human health. Clin TransI Med 2017;6(1):25. doi:10.1186/s40169-017-0153-6.

[65] Sun R, Wang X, Liu Y, Xia M. Dietary supplementation with fish oil alters the expression levels of proteins governing mitochondrial dynamics and prevents high-fat diet-induced endothelial dysfunction. Br J Nutr 2014;112(2):145-153. doi:10.1017/S0007114514000701.

[66] Sugasini D, Lokesh BR. Rats given linseed oil in microemulsion forms enriches the brain synaptic membrane with docosahexaenoic acid and enhances the neurotransmitter levels in the brain. Nutr Neurosci 2015;18(2):87-96. doi:10.1179/1476830514Y.0000000111.

[67] Arterburn LM, Hall EB, Oken H. Distribution, interconversion, and dose response of $n-3$ fatty acids in humans. Am J Clin Nutr 2006;83(6 Suppl):1467S-1476S.

[68] Brenna JT, Salem N Jr, Sinclair AJ, Cunnane SC, International Society for the Study of Fatty Acids and Lipids, ISSFAL. alpha-Linolenic acid supplementation and conversion to n-3 long-chain polyunsaturated fatty acids in humans. Prostaglandins Leukot Essent Fatty Acids 2009;80(2-3):85-91. doi:10.1016/j.plefa.2009.01.004.

[69] Plourde M, Cunnane SC. Extremely limited synthesis of long chain polyunsaturates in adults: implications for their dietary essentiality and use as supplements. Appl Physiol Nutr Metab 2007;32(4):619634. doi:10.1139/H07-034.

[70] Vermunt SH, Mensink RP, Simonis MM, Hornstra G. Effects of dietary alpha-linolenic acid on the conversion and oxidation of $13 \mathrm{C}$-alphalinolenic acid. Lipids 2000;35(2):137-142.

[71] Michael-Titus AT, Priestley JV. Omega-3 fatty acids and traumatic neurological injury: from neuroprotection to neuroplasticity? Trends Neurosci 2014;37(1):30-38. doi:10.1016/j.tins.2013.10.005

[72] Michael-Titus AT. Omega-3 fatty acids and neurological injury. Prostaglandins Leukot Essent Fatty Acids 2007;77(5-6):295-300 doi:10.1016/j.plefa.2007.10.021.

[73] Mills JD, Bailes JE, Sedney CL, Hutchins H, Sears B. Omega-3 fatty acid supplementation and reduction of traumatic axonal injury in a rodent head injury model. J Neurosurg 2011;114(1):77-84. doi:10.317 1/2010.5.JNS08914.

[74] Liu Q, Wu D, Ni N, Ren H, Luo C, He C, et al. Omega-3 polyunsaturated fatty acids protect neural progenitor cells against oxidative injury. Mar Drugs 2014;12(5):2341-2356. doi:10.3390/md12052341.

[75] Wu A, Ying Z, Gomez-Pinilla F. The salutary effects of DHA dietary supplementation on cognition, neuroplasticity, and membrane homeostasis after brain trauma. J Neurotrauma 2011;28(10):21132122. doi:10.1089/neu.2011.1872.

[76] Wu A, Ying Z, Gomez-Pinilla F. Omega-3 fatty acids supplementation restores mechanisms that maintain brain homeostasis in traumatic brain injury. J Neurotrauma 2007;24(10):1587-1595. doi:10.1089/ neu.2007.0313.

[77] Yin Y, Li E, Sun G, Yan HQ, Foley LM, Andrzejczuk LA, et al. Effects of DHA on hippocampal autophagy and lysosome function after traumatic brain injury. Mol Neurobiol 2017. doi:10.1007/s12035-0170504-8.

[78] Schober ME, Requena DF, Abdullah OM, Casper TC, Beachy J, Malleske D, et al. Dietary docosahexaenoic acid improves cognitive function, tissue sparing, and magnetic resonance imaging indices of edema and white matter injury in the immature rat after trau matic brain injury. J Neurotrauma 2016;33(4):390-402. doi:10.1089/ neu.2015.3945

[79] Mills JD, Hadley K, Bailes JE. Dietary supplementation with the omega-3 fatty acid docosahexaenoic acid in traumatic brain injury. Neurosurgery 2011;68(2):474-481; discussion 481. doi:10.1227/ 
NEU.0b013e3181ff692b.

[80] Bailes JE, Mills JD. Docosahexaenoic acid reduces traumatic axonal injury in a rodent head injury model. J Neurotrauma 2010;27(9):16171624. doi:10.1089/neu.2009.1239.

[81] Menard C, Patenaude C, Gagne AM, Massicotte G. AMPA receptormediated cell death is reduced by docosahexaenoic acid but not by eicosapentaenoic acid in area CA1 of hippocampal slice cultures. J Neurosci Res 2009;87(4):876-886. doi:10.1002/jnr.21916.

[82] Wu A, Ying Z, Gomez-Pinilla F. Dietary omega-3 fatty acids normalize BDNF levels, reduce oxidative damage, and counteract learning disability after traumatic brain injury in rats. J Neurotrauma 2004;21(10):1457-1467. doi:10.1089/neu.2004.21.1457.

[83] Lawrence DW, Sharma B. A review of the neuroprotective role of vitamin $D$ in traumatic brain injury with implications for supplementation post-concussion. Brain Inj 2016;30(8):960-968. doi:10.3109/02 699052.2016.1147081.

[84] Harms LR, Burne TH, Eyles DW, McGrath JJ. Vitamin D and the brain. Best Pract Res Clin Endocrinol Metab 2011;25(4):657-669. doi:10.1016/j.beem.2011.05.009.

[85] Tang H, Hua F, Wang J, Yousuf S, Atif F, Sayeed I, et al. Progesterone and vitamin D combination therapy modulates inflammatory response after traumatic brain injury. Brain Inj 2015:1-10. doi:10.3 109/02699052.2015.1035330.

[86] Tang H, Hua F, Wang J, et al. Progesterone and vitamin D: Improvement after traumatic brain injury in middle-aged rats. Horm Behav 2013;64(3):527-538.

[87] Cekic M, Sayeed I, Stein DG. Combination treatment with progesterone and vitamin $\mathrm{D}$ hormone may be more effective than monotherapy for nervous system injury and disease. Front Neuroendocrinol 2009;30(2):158-172. doi:10.1016/j.yfrne.2009.04.002.

[88] Pfeiffer CM, Sternberg MR, Schleicher RL, Haynes BM, Rybak ME, Pirkle JL. The CDC's Second National Report on Biochemical Indicators of Diet and Nutrition in the U.S. Population is a valuable tool for researchers and policy makers. J Nutr 2013;143(6):938S-947S. doi:10.3945/jn.112.172858.

[89] Hua F, Reiss JI, Tang H, Wang J, Fowler X, Sayeed I, et al. Progesterone and low-dose vitamin $\mathrm{D}$ hormone treatment enhances sparing of memory following traumatic brain injury. Horm Behav 2012;61(4):642-651. doi:10.1016/j.yhbeh.2012.02.017.

[90] Witham MD, Dove FJ, Sugden JA, Doney AS, Struthers AD. The effect of vitamin $D$ replacement on markers of vascular health in stroke patients - a randomised controlled trial. Nutr Metab Cardiovasc Dis 2012;22(10):864-870. doi:10.1016/j.numecd.2010.11.001.

[91] Berridge MJ. Vitamin D cell signalling in health and disease. Biochem Biophys Res Commun 2015;460(1):53-71. doi:10.1016/j.bbrc. 2015.01.008.

[92] Thomson AD, Guerrini I, Marshall EJ. The evolution and treatment of Korsakoff's syndrome: out of sight, out of mind? Neuropsychol Rev 2012;22(2):81-92. doi:10.1007/s11065-012-9196-z.

[93] Jhala SS, Hazell AS. Modeling neurodegenerative disease pathophysiology in thiamine deficiency: consequences of impaired oxidative metabolism. Neurochem Int 2011;58(3):248-260. doi:10.1016/j.neuint. 2010.11.019.

[94] Liu D, Ke Z, Luo J. Thiamine deficiency and neurodegeneration: the interplay among oxidative stress, endoplasmic reticulum stress, and autophagy. Mol Neurobiol 2017;54(7):5440-5448. doi:10.1007/ s12035-016-0079-9.

[95] Shear DA, Dixon CE, Bramlett HM, Mondello S, Dietrich WD, DengBryant $Y$, et al. Nicotinamide treatment in traumatic brain injury: operation brain trauma therapy. J Neurotrauma 2016;33(6):523-37. doi:10.1089/neu.2015.4115.

[96] Maiese K, Chong ZZ. Nicotinamide: necessary nutrient emerges as a novel cytoprotectant for the brain. Trends Pharmacol Sci 2003;24(5):228-232. doi:10.1016/S0165-6147(03)00078-6.

[97] Kristian T, Balan I, Schuh R, Onken M. Mitochondrial dysfunction and nicotinamide dinucleotide catabolism as mechanisms of cell death and promising targets for neuroprotection. J Neurosci Res 2011;89(12):1946-1955. doi:10.1002/jnr.22626.

[98] Buratti P, Gammella E, Rybinska I, Cairo G, Recalcati S. Recent advances in iron metabolism: relevance for health, exercise, and performance. Med Sci Sports Exerc 2015;47(8):1596-1604. doi:10.1249/ MSS.0000000000000593.

[99] Scott SP, Murray-Kolb LE. Iron status is associated with performance on executive functioning tasks in nonanemic young women. J Nutr 2016;146(1):30-37. doi:10.3945/jn.115.223586.

[100] Winter WE, Bazydlo LA, Harris NS. The molecular biology of human iron metabolism. Lab Med 2014;45(2):92-102. doi:10.1309/LMF28S2GIMXNWHMM.

[101] Hong CT, Huang YH, Liu HY, Chiou HY, Chan L, Chien LN. Newly diagnosed anemia increases risk of parkinson's disease: a populationbased cohort Study. Sci Rep 2016;6:29651. doi:10.1038/srep29651.

[102] Nelson C, Erikson K, Pinero DJ, Beard JL. In vivo dopamine metabolism is altered in iron-deficient anemic rats. J Nutr 1997;127(12):22822288.

[103] Iverson GL, Gardner AJ, Terry DP, Ponsford JL, Sills AK, Broshek DK, et al. Predictors of clinical recovery from concussion: a systematic review. Br J Sports Med 2017;51(12):941-948. doi:10.1136/bjsports-2017-097729.

[104] Gardner A, Iverson GL, Levi CR, Schofield PW, Kay-Lambkin F Kohler RM, et al. A systematic review of concussion in rugby league. Br J Sports Med 2015;49(8):495-498. doi:10.1136/bjsports-2013-093102.

[105] Black AM, Sergio LE, Macpherson AK. The epidemiology of concussions: number and nature of concussions and time to recovery among female and male canadian varsity athletes 2008 to 2011. Clin J Sport Med 2017;27(1):52-56. doi:10.1097/JSM.0000000000000308.

[106] Covassin T, Elbin RJ, Bleecker A, Lipchik A, Kontos AP. Are there differences in neurocognitive function and symptoms between male and female soccer players after concussions? Am J Sports Med 2013;41(12):2890-2895. doi:10.1177/0363546513509962.

[107] Eckner JT, Oh YK, Joshi MS, Richardson JK, Ashton-Miller JA. Effect of neck muscle strength and anticipatory cervical muscle activation on the kinematic response of the head to impulsive loads. Am J Sports Med 2014;42(3):566-576. doi:10.1177/0363546513517869.

[108] Kroshus E, Baugh CM, Stein CJ, Austin SB, Calzo JP. Concussion reporting, sex, and conformity to traditional gender norms in young adults. J Adolesc 2017;54:110-119. doi:10.1016/j.adolescence.2016.11.002

[109] Ono KE. Sex-based differences as a predictor of recovery trajectories in young athletes after a sports-related concussion: response. Am J Sports Med 2016;44(6):NP31. doi:10.1177/0363546516651048.

[110] Reynolds BB, Patrie J, Henry EJ, Goodkin HP, Broshek DK, Wintermark $\mathrm{M}$, et al. Effects of sex and event type on head impact in collegiate soccer. Orthop J Sports Med 2017;5(4):2325967117701708. doi:10.1177/2325967117701708.

[111] Zuckerman SL, Apple RP, Odom MJ, Lee YM, Solomon GS, Sills AK. Effect of sex on symptoms and return to baseline in sport-related concussion. J Neurosurg Pediatr 2014;13(1):72-81. doi:10.3171/20 13.9.PEDS13257.

[112] Sato A, Shimoyama Y, Ishikawa T, Murayama N. Dietary thiamin and riboflavin intake and blood thiamin and riboflavin concentrations in college swimmers undergoing intensive training. Int J Sport Nutr Exerc Metab 2011;21(3):195-204. doi:10.1123/ijsnem.21.3.195.

[113] Parks RB, Hetzel SJ, Brooks MA. Iron deficiency and anemia among collegiate athletes: a retrospective chart review. Med Sci Sports Exerc 2017;49(8):1711-1715. doi:10.1249/MSS.0000000000001259.

[114] Sandstrom G, Rodjer S, Jacobsson S, Nelson D, Borjesson M. Increased level of serum hepcidin in female adolescent athletes. Clin J Sport Med 2017. doi:10.1097/JSM.0000000000000423.

[115] Petkus DL, Murray-Kolb LE, De Souza MJ. The unexplored crossroads of the female athlete triad and iron deficiency: a narrative review. Sports Med 2017;47(9):1721-1737. doi:10.1007/s40279-017-0706-2. 